\title{
Negative findings
}

\author{
Fiona Godlee editor, BMJ
}

Patients about to undergo surgery for cancer will want to know that everything possible is being done to help their immune system fight against a recurrence. They and their surgeons are up against the immunosuppressive effects of surgery itself, general anaesthesia, and post-operative opioids. Various animal and human studies over the past 40 years have raised hopes that regional anaesthesia could help by reducing the response to surgical stress and the need for opioids. It's a nice idea. Sadly the evidence against it being true is mounting. In this week's $B M J$, Paul Myles and colleagues report the long term follow up of their randomised trial of post-operative epidural block in patients undergoing potentially curative abdominal surgery for cancer (doi:10.1136/bmj.d1491). They found no effect on cancer free survival.

The authors are clear about the study's limitations. Patients in both groups had general anaesthesia and opioids, and the study included a mix of cancers, which means they couldn't look for cancer specific effects. But as B C H Tsui and J S Green say in their linked editorial (doi:10.1136/bmj.d1605), the study was powered to detect a difference in recurrence-free survival greater than $30 \%$, similar to the difference reported in a recent, much quoted retrospective study in women with breast cancer. "An effect of this size would surely have been proved during the past 30 years of research and seems too good to be true," they say.

Tsui and Green see obstacles to further research in this area. Much larger studies will be needed to find a small but still clinically important effect. They see hope in careful accrual of knowledge on the immunosuppressive effects of different anaesthetic and analgesic agents. Someone may yet find an optimal combination of drug and regional technique that will improve patients' survival.

There's one other thing to note about this study: the methodology included subgroup analyses. And so, you will be asking (as good disciples of the CONSORT reporting guidelines): were these prespecified or were they post hoc and exploratory? The answer is that some were prespecified and some were exploratory, as clearly stated in the methods section in the full report on bmj.com. Analyses that are specified in the trial protocol are more reliable. Multiple exploratory analyses after the primary outcome has proved negative are bound to throw up false positive results in one subgroup or other.

Sadly not all trials are this well reported, and yet again industry funded trials have been found to fall seriously short. In their systematic review, Xin Sun and colleagues show a clear association between industry funding and the misreporting of subgroup analyses (doi:10.1136/bmj.d1569). They looked at 1140 reports of randomised trials published in 2007 . Where there was no statistically significant difference in the primary outcome, industry funded trials were more likely to report subgroup analyses than non-industry funded trials. And they were less likely to pre-specify their subgroup hypotheses or to test for interaction between subgroups. Whether they knew what they were doing (or not doing) is known only to themselves.

Cite this as: $B M J 2011 ; 342: \mathrm{d} 2053$ 